\title{
Role of Polyphenols like Resveratrol in Cancer Prevention and Treatment-Especially in Combination with Other Polyphenols Like Quercetin-Detailed Mode of Action and Future Perspectives-A Systematic Review
}

\author{
Kulvinder Kochar Kaur ${ }^{1 *}$, Gautam Allahbadia ${ }^{2}$ and Mandeep Singh ${ }^{3}$ \\ ${ }^{1}$ Scientific Director, DR Kulvinder Kaur Centre for Human Reproduction, Jalandhar, \\ Punjab, India \\ ${ }^{2}$ Scientific Director, Rotunda-A Centre for Human Reproduction, Mumbai, India \\ ${ }^{3}$ Consultant Neurologist, Swami Satyanand Hospital, Jalandhar, Punjab, India \\ *Corresponding Author: Kulvinder Kochar Kaur, Scientific Director, DR Kulvinder \\ Kaur Centre for Human Reproduction, Jalandhar, Punjab, India.
}

\author{
Received: March 17, 2020 \\ Published: June 08, 2020 \\ (C) All rights are reserved by Kulvinder \\ Kochar Kaur., et al.
}

\begin{abstract}
Earlier having reviewed the role of resveratrol,a polyphenol along with other polyphenols in obesity,DM, autoimmune disease and role of programmed death-1(PD1)/programmed death-ligand1(PD-L1) system in ovarian and other cancers here we decided to carry out a systematic review on role of resveratrol,alone or in combination with other polyphenols in prevention as well as therapy of various cancers like ovarian cancer,breast cancer especially basal cell and HER+ve, besides multiple melanoma, colorectal and other cancers on the basis of senescent cell approach in cancer cells.We found thatPolyphenols inhibit senescent-associated secretory phenotype(SASP) factors secretion from senescent cells to form anti-tumor microenvironment for avoiding cancer.Once tumorigenesis starts, polyphenols are capable of inhibiting cancer by using oncogenes-oxidative stress, DNA Damage response(DDR) as well as endoplasmic reticulum(ER) stress induced cancer senescence. Concomitantly using 2 Polyphenols or a mixture of Polyphenols as well as other anti- cancer drugs increase therapeutic efficacy.This combined use of Polyphenols will be critical in future for this purpose in cancer prevention as well as therapy.

Keywords: Resveratrol; Polyphenol; Ovarian Cancer; Programmed Death-1(PD1)/Programmed Death-Ligand1 (PD-L1);Breast Cancer; SASP Factors; DDR; SASP Factors
\end{abstract}

\section{Introduction}

Earlier we had reviewed the management of ovarian carcinomaalong with role of PD1-PD-L1 pathway in ovarian carcinoma and role of resveratrol in chronic inflammatory diseases was autoimmune diseases as well as importance of immunometabolism and NK Cells function in obesity-correlating with comorbidities development like cancer and type 2diabetes [1-5]. Here we decided to further review the role of natural polyphenols like Resveratrol (RES) in various cancers along with its mechanism of action involvingPD1-PD-L1 pathway as well as Immunometabolism in curing or aiding prevention of drug resistance development besides ovarian cancers, and other cancers like certain breast cancers.

\section{Methods}

We carried out a Pubmed Search using the MeSH terms like polyphenols like Resveratrol (RES);other polyphenols; PD1-PD-L1 pathway; immunometabolism with role in treating various cancers with resveratrol or other polyphenol combinations.

\section{Results and Discussion}

We found a total of 1328 articles out of which we selected 50 articles for this review. No meta-analysis was done.
Ovarian carcinoma is 1 out of 3 commonest gynaecological malignancies having highest mortality and is in $5^{\text {th }}$ place regarding cancer associated deaths out of 10 main human cancers in women world over [6].Right now the initial option with the angle to cure is surgical removal with adjuvant chemotherapy or radiotherapy [7]. But with intrinsic side effects, massive cost as well as primary along with acquired drug resistance, the clinical results are hardly satisfactory [8]. Hence use of innovative, low toxic, very effective anti-tumour drugs or chemotherapy resensitizing compounds is key for general improvement regarding prognosis of Ovarian carcinoma patients.

Resveratrol (RES) is a nonflavonoid polyphenol that has stilbene structural elements and has been widely found in lot of plants like grapes as well as lilies [9].The medicinal impact of RES has been shown in antiinflammation, antimicrobial, anti-oxidant, antiviral, anti-atherosclerotic, immunomodulatory as well as neuroprotection. Importantly, lot of proof has collected regarding antitumor properties of RES against various cancers with a lot of signaling pathways that give its mode of action regarding antiproliferative as well as apoptosis inducing actions brought about by RES. Therapeutic actions have been shown in Hepatocellular Carci- 
noma (HCC), Ovarian carcinoma as well as leukemia. RES was initially shown to suppress hepatoma cell invasion independently of its antiproliferative actions [10].Opipari.,et al. demonstrated RES causes autophagocytosis in Ovarian cancer cells [6]. Cancer chemopreventive actions of RES in promyelocytic leukemia cells was demonstrated by Jang.,et al. for the $1^{\text {st }}$ instance [11]. More work have tested the potential of RES in inducing cell apoptosis in Ovarian cancer cells [12]. But the probability of RES in immune activation as well as promotion of immunogenic cell death (ICD) in this scenario has not been defined.

Recently emerging;proof showed that certain platinum based chemical drugs, like oxaliplatin, stimulated ICD besides cell apoptosis in cancer cells [13]. Usually, 3 major signal cues have been released when host cells undergo ICD, that include cell surface exposure of calreticulin (CRT) that stimulates phagocytosis of dendritic cells (DC's). ATP release that recruits DC's, as well as secretion of high mobility group box1 (HMGB1) that promotes complexation of DC cells with moribund tumor cells as well as stimulates T cells based particular tumor immunity [14]. Subsequently ICD brings about DC maturation as well as infiltration of cytotoxic $\mathrm{T}$ cells into tumor mass that gives chance for combinational therapy with immune checkpoint inhibitor like PD-L1 as well as PD-1 antibodies [15].

Zhang.,etal. tried to study if ICD was part of the antitumor action of RES against Ovarian carcinoma. RES suppressed cell proliferationas well as causedapoptosis in Ovarian carcinoma cells. Additionally,RES treatment stimulated cell surface exposure of CRT, HMGB1 secretion along with ATP release. Vaccination with RES-pretreated ID-8 cells markedly inhibited growth of later inoculated xenograft tumor. Direct delivery of RES suppressed tumor propagation associated with compromised cell proliferation along with escalated cells apoptosis. They further characterized escalation of mature DC's, as well as cytotoxic T cells in xenograft tumor in response to RES treatment, that also inhibited transforming growth factor beta (TGF $\beta$ ) synthesis as well as a stimulation ofboth IL $12 \mathrm{p} 7$ as well as interferon gamma (IFNy) secretion. What was of maximum importance, they showed that combination with PD-1 antibody markedly inhibited tumor growth where as depleting CD8+ $\mathrm{T}$ cells by neutralizing antibodies restored xenograft propagation. Thus, their data pointed that RES caused antitumor action against Ovarian carcinoma by both apoptosis along with ICD pathways [16].

Unlike immunologically "hot tumors like lung cancer, melanoma along with bladder cancer, most breast carcinomas are not inherently immunogenic. Thus, they classically show low $\mathrm{T}$ cell infiltrationand are not likely to improve with immune checkpoint inhibitor therapies [17]. Basal-like breast carcinomas are different in this matter of immunologically "cold' [18] as well as HER2 positive [19] subtypes, both of which exhibit proof of immunogenicity as well as tumor immune infiltratesas well as stromal and intratumoral tumor infiltrating lymphocytes i.e. a good predictive marker regarding response toimmunotherapy. In relation to this, the usu- ally rare expression in most breast carcinomas of programmed death ligand-1 (PD-L1) i.e. a prototypical immunosuppressive molecule on cancer cells which engages its receptor PD-1 on T cells for repressing $\mathrm{T}$ cell mediated immune surveillance [20], is significantly high in basal like as well as HER2 positive tumors, thus indicating that PD-L1 confers a survival benefitin the tumor microenvironment (TME) of these particular breast cancer subtypes. But the response rates documented in clinical trials for breast cancer with PD-1/PD-L1check point inhibitor as single drugs have been very disappointing (5-10\%), although better clinical action (upto30\%) as well as durable overall responses have been seen in patients with Basal-like/HER2 positive breastcancer as well as positive expression ofPD-L1 [21]. As per this, the finding of innovative strategies for blocking the immune inhibitory signal of PD-L1in Basal-like as well as HER2 positive breast cancer subtypes is urgently required.

Recently, the field of cancer immunometabolism has been giving us understanding of the key role of metabolism in regulating immune cell function [22]. A lot is known now how phenotypic switching of $\mathrm{T}$ cells can be efficacious against tumor cells, essentially needs metabolic specialization, as well as how particular metabolic actions as well as tumor driven switch in the abundance of particular metabolites shape local immunosuppression as well as decrease the metabolic fitness of tumor infiltrating lymphocytes. Actually, the inhibitory nature of the metabolic cross talk among tumor as well as immune cells in the TME supports its acting as a target for overcoming the escape of cancer cells along with overcome immunotherapy resistance. Likewise, different combinations of immunotherapies using metabolic agents had the objective of rewiring $\mathrm{T}$ cell fitness, by repressing the mimmunosuppressive metabolic traits within the TME are getting tested in clinical trials [23]. Still the cancer cell-autonomous metabolic reprogramming in the form of real driver of immune checkpoint signaling with in tumor cells has been totally neglected in cancer immunometabolism. Actually, we don't even have slightest insight into how pharmacological manipulations targeting core metabolic checkpoint like AMP activated protein kinase (AMPK), mammalian target of rapamycin (mTOR), as well as silent information regulator 1 (SIRT1), may fine tune the expression of immune checkpoint receptors in cancer cells.

Thus,Verdura,etal. while trying to explore the PD-L1targeted effects of metabolism targeting agents with separate modes of action, exposure to the dietary polyphenol Resveratrol (PD-L1) showed the separate ability to develop a distinct PD-L1 electrophoretic migration pattern. Utilizing biochemical assays, computer-aided docking/molecular dynamic simulations as well as fluorescent microscopy, they observed that RES can act as a direct inhibitor of glyco-PD-L1-processing enzymes ( $\alpha$-glucosidase/ $\alpha$-mannosidase) which modulates N-linked glycan decoration of PD-L1, thus promoting the ER retention of a mannose rich, abnormally glycosylated form of PD-L1. RES was also predicted to interact with the inner surface of PD-L1 involved in the interaction with PD-1, almost accurately occupying the target space ofthe small compound BMS-202 which binds to as well as induces dimerizationof PD-L1. The capacity of RES to directly target PD-L1interferes with its stability as well 
as trafficking, ultimately interfering with its targeting to the cancer cell plasma membrane. Impedance based real time cell-analysis (xCELLigence) demonstrated that cytotoxic T lymphocyte activity, was importantly exaggerated when cancer cells were previously exposed to RES. This unforeseen immunomodulating action of RES might highlight new strategies for restoring $\mathrm{T}$ cell function by targeting thePD-1/PD-L1 immunologic checkpoint with the use of natural polyphenols(Figure 1) [24].

\section{Senescent cell characteristics}

3 phases at culture-phase I-period of slow proliferation prior to $1^{\text {st }}$ confluent sheet conformation; phase II-during whichsubcultivation is needed for rapid cell proliferation; phase III-cell culture finishes with permanent loss of proliferation potential of cells(Figure 1) [25]. Hayflick posited limited lifetime of diploid cell lines in vitro might indicate aging or senescence at cellular level.

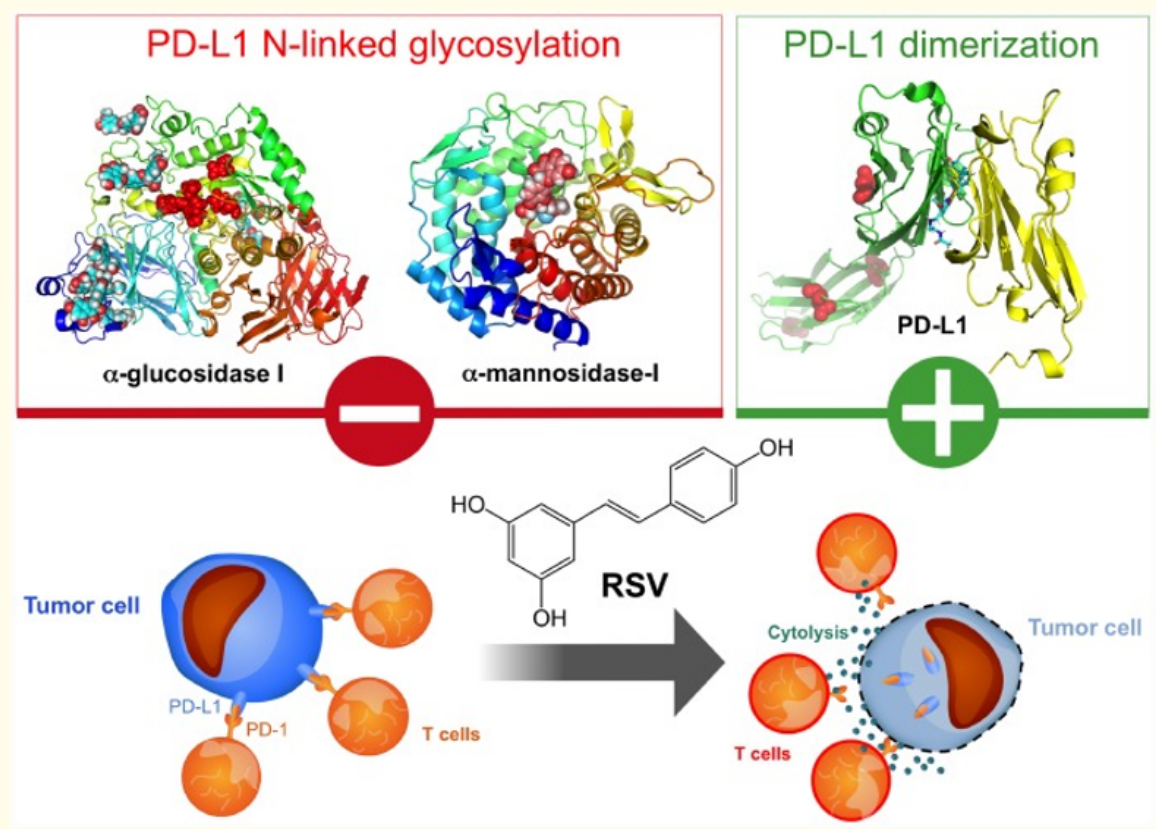

Figure 1: Courtesy reference number [24]: Resveratrol enhances antitumor T cell immunity by promoting abnormal glycosylation and dimerization of PD-L1. Post-translational modifications such as glycosylation, phosphorylation, palmitoylation or ubiquitination are essential for the folding, intracellular transport, and stabilization of the PD-L1 protein PD-L1 is highly glycosylated, and N-linked glycosylation of PD-L1 critically maintains its protein stability and is required for its interaction with PD-1 to efficiently suppress T-cell activity. Recently, Bristol-Myers Squibb (BMS)-developed compounds with a common (2-methyl-4-biphenylyl)methanol scaffold have been reported to block the PD-1/PD-L1 interaction by interacting with the cavity formed by the two PD-L1 monomers and inducing the dimerization of PD-L1Structural studies have revealed a dimeric protein complex with a single small molecule that stabilizes the dimer and thereby occludes the PD-1 interaction surface of PD-L1. We propose that RSV is a naturally occurring, double-strike PD-1/PD-L1 immune checkpoint inhibitor capable of directly blocking the enzymatic machinery in charge of the N-linked glycosylation of the nascent PD-L1 at the endoplasmic reticulum or directly binding to PD-L1 surfaces to induce PD-L1 dimerization and block PD-1 binding. This unforeseen ability of RSV to directly interfere with PD-L1 stability and trafficking impedes the correct targeting of PD-L1 to the cancer cell plasma membrane and ultimately elicits drastically enhanced cytotoxic T-lymphocyte immune-surveillance against tumor cells. These findings might illuminate new approaches to restore T-cell function by targeting the PD-1/PD-L1 immunologic checkpoint with natural polyphenols.

This senescence that is correlated with telomere loss or dysfunction is known as "replicative senescence". Without telomere shortening senescence induced by certain stimuli-as it occurs prior totelomere shortening-known as premature(Figure 2) [26]. Later cell senescence in vivo-like mouse embryo mesonephros, endolymphatic sac of inner earas well as apical ectodermal ridgeof the limbs;human maternal-face interface [27],human Rheumatoid Arthritis tissues, human skin, discas well as lung. Common features of Polyphenoli) enlarged as well as irregular shaped cell body ii) increased action of SA- $\beta$ gal, as well as iii) cell cycle arrest. Senescent state cells-accompanied by a) formation of senescence-asso- ciated heterochromatin foci (SAHF's), b) lack of Ki 67 that is associatedwith cell cycle, c) induction of DNA damage response (DDR) d) activation of p53/p21 as well as p16/Rb signaling pathway [28]. Apoptotic resistanceis widely seenthat might be due to inactivation of p53 as well as an upregulation ofApoptotic B-cell lymphoma-2 (BCL-2) in senescent human fibroblasts as well as keratinocytes [29]. But senescent endothelial cells display increase sensitivity to that of Apoptotic with decreased BCL-2 as well as increased BCL2-associated X apoptosis regulator (BAX) expression [30]. Senescent cells secrete -series of bioactive molecules called senescentassociated secretory phenotype (SASP) [31], Secreted molecules 

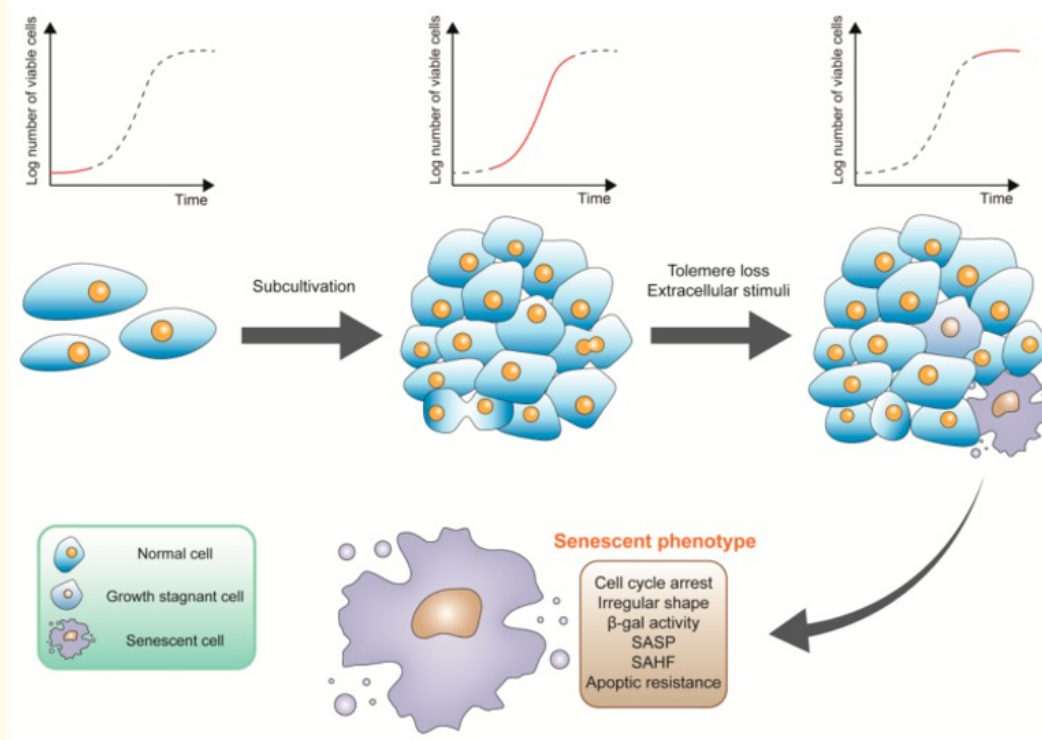

Figure 2: Courtesy reference number [50]: Process and phenotype of senescence. Cell growth curve, senescence process, and characteristics of senescent cells are displayed. SASP; senescence-associated secretory phenotype SAHF, senescence-associated heterochromatin foci.

stimulate proinflammatory cytokines, like interleukin (IL)-6, IL-8. chemokines, growth factors, as well as matrix modeling enzymes can cause series of physiological functions like senescence promotion of adjacent cells in tissue microenvironment [28].

\section{Polyphenol sources}

Plenty-ellagic acid, gallic acid, tannic acid 3. as well as capsaicin are usual phenolic acids present in a wide variety of plant derived foods like teas, tropical fruits, soy as well as wheat products [32]. Resveratrol (3, 4'5'-trihydroxy stilbene) -representative member of stilbene family -popular due to explanation of "French paradox". Abundant in grape skin, peanuts as well as redwine [33]. Lignansphytooestrogens-widely seen in Schisandraceae plants-other edible plants like flaxseed, cereal products, brassica vegetables [34]. Flavonoids -secondary metabolites in plant as well as fungus -high amount in fruits vegetables legumes, teas, dark chocolates [35]

Synergistic effects of polyphenol administration

See figure 3 combined resveratrol along with quercetin, 2Polyphenols markedly aided the degree of Senescent cells as compared to each single cell used in culture glioma cells [36]. ii) strengthened anti-cancer effect of usual regimens of chemotherapy as well as radiotherapy. RES increased Senescence caused by temozolomide as well as further decreased temozolomide drug resistance in cancer cells in glioma cells [37]. Also, RES could resensitize paclitaxel resistant cancer cells to paclitaxel [38] as well as overcome gefitinib resistance of non-small lung cancer by incgefitinib concentration when used together with gefitinib [39]. Also, RES treatment DNA double stranded breaks as well as reactive oxygen species (ROS) production for reinforcing ionizing radiation-induced premature senescence along with a decreased radioresistance of lung cancer cells [40].
Molecular mechanisms of polyphenols in cancer treatment

i) Inhibit proliferation as well asmigration ii) cancer cell apoptosis induction iii) impeding immunosuppression etc. Like RES inhibited proliferation along with induced apoptosis of human multiple myeloma cell lines by downregulating different proliferative as well as anti-apoptotic gene products like cyclin D1, cIAP-2, X-linked inhibitor of apoptosis protein (XIAP), surviving, B-cl2, B-cl-Xl, Bfl-1/ A1 as well as TNF receptor associated factor2 (TRAF 2) [41]. A representating flavonoid quercetin-directly binds to PI3K for inhibiting PI3K/AKT signaling pathway as well as inhibit H-RAS - induced invasion as well as migration in MCF10A human epithelial cells [42]. Action of RES already discussed in ref 24 on PD1/PDL1.

\section{Targeting tumor microenvironment for cancer prevention}

Senescent cells collect with age -producing lots of SASP factors that may become harmfulif unchecked. Like fibroblasts are main cell kinds in tissue stroma and get involved in remodeling as well as repair processes, On being Senescent, they express SASP factors that simulate that of fibroblasts next to some carcinomas, causing a tumor favouring microenvironment and favour proliferation of myeloma cells as well as colorectal cancer formation [43]. Targeting SASP for inducing anti-tumor microenvironment might aid in cancer prevention. Chronic RES treatment decreased the secretion of SASP factors in Senescent MRC5 fibroblasts, thus ameliorating intra cellular signaling like phosphorylation of extra cellular signal -regulated kinase (ERK) as well as $\mathrm{S6}$ proteins in endothelial colony forming cells for decreased angiogenesis as well as inhibited epithelial to mesenchymal transition, proliferation as well as invasiveness of myeloma cells respectively(Figure4) [44]. A plant flavone apigenin significantly inhibited SASP secretion profile of human fibroblasts cells by blunting IкB expression, thereby decreased the aggressive phenotype of human breast cancer cells as well as oral 


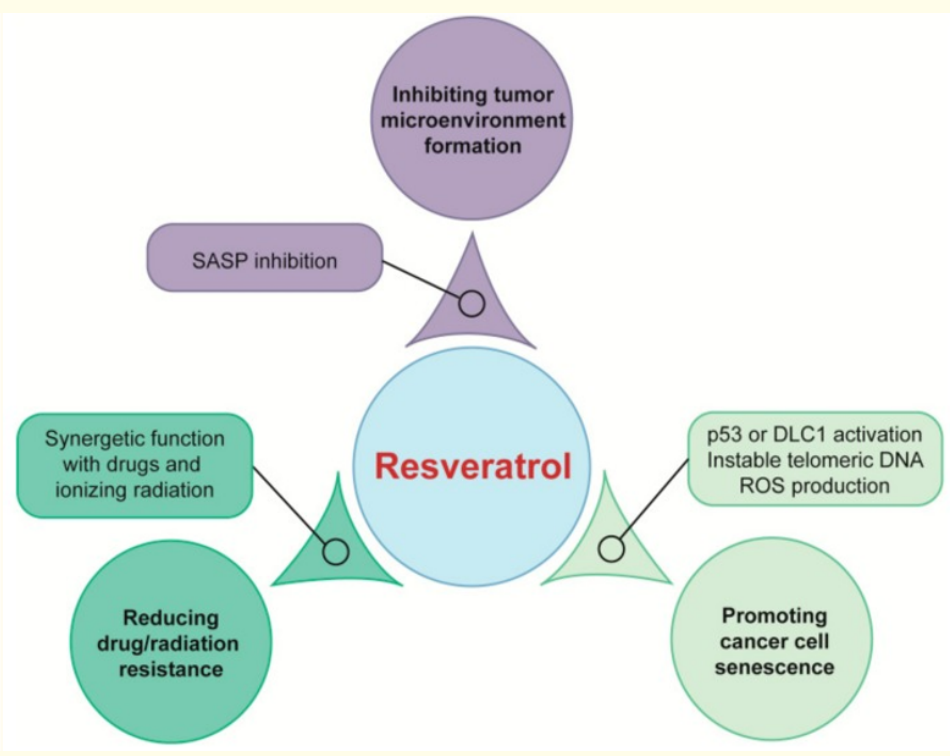

Figure 3: Courtesy reference number [50]: Potential functions of resveratrol in anti-tumor therapy. Resveratrol inhibit tumor microenvironment for cancer prevention, reduce drug/radiation resistance and induce cancer cell senescence for cancer therapy. SASP, senescence-associated secretory phenotype; DLC1, deleted in liver cancer1; ROS, reactive oxygen species.
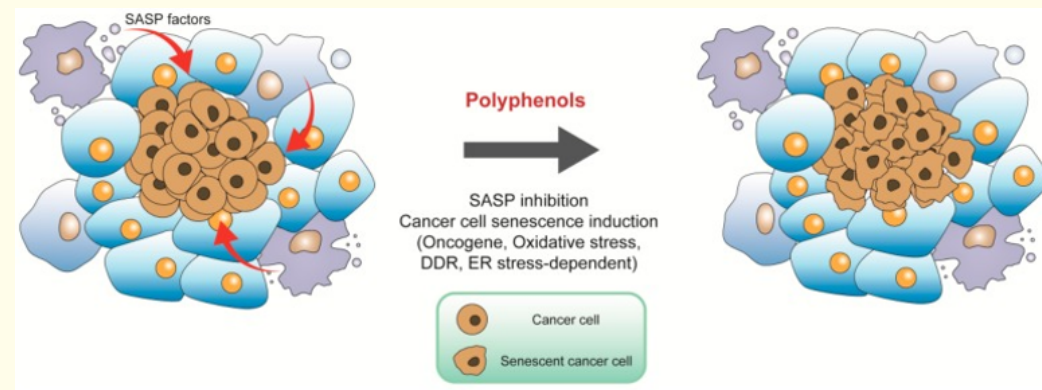

Figure 4: Courtesy reference number [50]: Roles of polyphenols in tumor suppression. Polyphenols inhibit secretion factors from senescent cells to impair tumor microenvironment and promote oncogene-, oxidative stress-, DNA damage response (DDR) and endoplasmic reticulum (ER) stress-induced cancer cell senescence.

administration of apigenin significantly inhibited SASP in kidneys of aged rats [45]. Resveratrol Increased expression of silent information regulator 1 (SIRT1) to decreased SASP as well as inhibited age -based spontaneous tumorigenesis in annual fish Nothobranchius guentheri [46]. Hence natural Polyphenols are potential in targeting tumor microenvironment for cancer avoidance(Figure 4).

\section{Oncogene induced senescence (OIS)}

Premature Senescence that gets induced by Oncogenes is known as OIS. OIS was primitively seen in 1997 when Oncogene RAS was introduced in primary human diploid fibroblasts, Oncogene RAS can transform primary cells into tumorigenic state in the case of tumor suppressors inactivation or the presence of cooperating Oncogene. But expression of Oncogene RAS ended in permanent G1 arrest accompanied with collection of p53 as well as p16 in primary rodent or human cells [47]. Other Oncogenes or tumor suppressor genes that are capable of inducing Senescence have been found since then. Oncogenes like RAS induced Senescence are commonly found in cancer cells. Many Polyphenols exert their action through OIS (Figure 4 as well as figure 5). Like suppression of RAS/Mitogen activated protein kinase (MAPK)/ERK as well asPI3K/AKTsignaling pathwaymediated via quercetin aided in glioma cell Senescence [48]. p53 as well as p16 are crucial molecules involved in senescence-induction. p53 activation was needed for RES to upregulateC-X-C motif chemokine receptor 2 (CXCR2) to drive cancer cells senescence and protect the cells from apoptosis (Figure 3) [49]. See figure 5 for overall actions of Polyphenols like stilbenes, flavonoids as well as phenolic acids [50].

\section{Conclusion}

Polyphenols are ubiquitous in our diet, being essentialsource of anti- cancer compounds. Targeting senescence is coming out as an anti- cancer strategywith Polyphenols having a role in senescence control. This review highlights important aspects of modes of Polyphenols in targeting senescence in both cancer prevention as well 


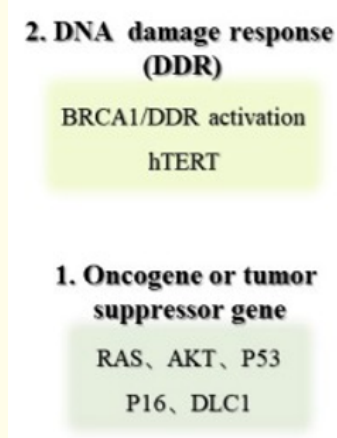

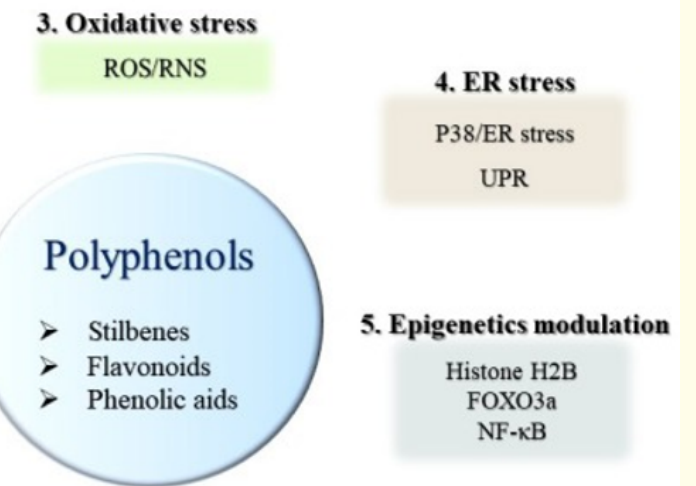
6. Other mechanisms SIRT1 Rictor/RhoA-GTPase

Figure 5: Courtesy reference number [50]: Molecular targets of polyphenols in senescence induction. Polyphenols can induce cancer cell senescence via various pathways, including (1) regulation of oncogene or tumor suppressor gene expression, (2) activation of DDR,

(3) promotion of oxidative stress, (4) induction of ER stress, (5) modulation of epigenetics, and (6) other mechanisms. AKT, protein kinase B; DLC1, deleted in liver cancer1; hTERT, human telomerase reverse transcriptase; ROS, reactive oxygen species; RNS, reactive nitrogen species; ER stress, endoplasmic reticulum stress; UPR, unfolded protein response; FOXO3a, forkhead box $03 a$; NF- $\kappa B$, nuclear factor kappa-light-chain-enhancer of activated B cells.

as therapy. Finally,Polyphenols inhibit SASP factors secretion from senescent cells to form anti-tumor microenvironment for avoiding cancer. Once tumorigenesis starts, Polyphenols are capable of inhibiting cancer by using Oncogenes-oxidative stress, DDR as well as ER stressinduced cancer senescence (Figure 4 as well as 5). Concomitantly using 2 Polyphenols or a mixture of Polyphenols as well as other anti-cancer drugs increase therapeutic efficacy. This combined use of Polyphenols will be critical in future for this purpose in cancer prevention as well as therapy.

\section{Bibliography}

1. Kulvinder Kochar Kaur., et al. "Advances in the Therapy of Advanced Ovarian Cancer-Special Emphasis on the PD1/PDL1 Pathway".Current Trends in Biomedical Engineering and Biosciences (2016): 001-003.

2. Kulvinder Kochar Kaur., et al. "An Update on High Grade Serous Ovarian Carcinoma - A Comprehensive Review". Acta Scientific Cancer Biology 3.3(2019): 23-35.

3. Kulvinder Kochar Kaur.,et al. "Alteration in Natural Killer (NK) cell Function inObesity-correlating with comorbidities development like cancer and type 2diabetes-A minireview". Journal of Endocrinology 3.2 (2019): 000140.

4. Kulvinder Kochar Kaur., et al. "Will Utilization of Resveratrol's Effects be Practical in Multiple Chronic Inflammatory Diseasesand Autoimmune Diseases: A Detailed Review of its Immune Responses and Further Clinical Development in $\mathrm{Hu}-$ mans in Future - ASystematic Review". Acta Scientific Microbiology Special1 (2019): 14-23.
5. Kulvinder Kochar Kaur., et al. "Role of Nutrients Competition in Immunometabolism - Effects on Immune Responses - A Systematic Review". Acta Scientific Nutritional Health 3.10 (2019): 197-204.

6. Torre LA., et al. "Ovarian cancer statistics,2018".CA: A Cancer Journal for Clinicians 68.4 (2018):284-296.

7. Fujiwara K., et al. "Paradigm shift in the management strategy for epithelial Ovarian cancer".American Society of Clinical Oncology Educational book 35 (2016):e247-e257.

8. Agarwal R and Kaye SB. "Ovarian cancer:strategies for overcoming resistanceto chemotherapy".Nature Reviews Cancer 3.7 (2003):502-516.

9. Nawaz Z., et al. "Therapeutic versatility of resveratrol derivatives".Nutrients 9.11 (2017).

10. Kozuki Y., et al. "Resveratrol suppresses hepatoma cell invasion independently of its antiproliferative action".Cancer Letters 167.2 (2001):151-156.

11. Opipari AW Jr., et al. "Resveratrol inducedautophagocytosis in Ovarian cancer cells". Cancer Research 64.2 (2004):696-703.

12. JangM., et al. "Cancerchemopreventive activity of resveratrol,a natural product derived from grapes".Science275.5297 (1997):218-220.

13. Gwak H.,et al. "ER stress mediated apoptosis by disruptingNlinked glycosylation of proteins in Ovarian cancer cells". Cancer Letters 371.2 (2016):347-353. 
14. Showalter A., et al. "Cytokines inimmunogenic cell death :application for Cancer immunotherapy".Cytokines 97 (2017):123-132.

15. PfieschkeC., et al. "Immunogenic chemotherapy:sensitizes tumors to check point blockade therapy".Immunity 44.2 (2016):343-354

16. Zhang Y., et al. "Resveratrol induces Immunogenic cell death of human and murine Ovarian carcinoma cells".Infectious Agents and Cancer14 (2019):27.

17. Chrétien S., et al. "Beyond PD-1/PD-L1 Inhibition: What the Future Holds for Breast Cancer Immunotherapy". Cancers (Basel) 11 (2019):E628.

18. Ali HR., et al. "PD-L1 protein expression in breast cancer is rare, enriched in basal-like tumours and associated with infiltrating lymphocytes". Annals of Oncology 26 (2015):14881493.

19. Griguolo G., et al. "Interaction of host immunity with HER2targeted treatment and tumor heterogeneity in HER2-positive breast cancer". Journal for ImmunoTherapy of Cancer 7 (2019):90.

20. Yao S., et al. "Advances in targeting cell surface signalling molecules for immune modulation". Nature Reviews Drug Discovery 12 (2013):130-146.

21. Adams S., et al. "Pembrolizumab monotherapy for previously untreated, PD-L1-positive, metastatic triple-negative breast cancer: cohort B of the phase II KEYNOTE-086 study". Annals of Oncology 30 (2019):405-411.

22. Li X., et al. "Navigating metabolic pathways to enhance antitumour immunity and immunotherapy". Nature Reviews Clinical Oncology 16 (2019):425-441.

23. Guo C., et al. "Immunometabolism: A new target for improving cancer immunotherapy". Advances in Cancer Research 143 (2019):195-253.

24. Verdura S., et al. "Resveratrol targets PD-L1 glycosylation and dimerization to enhance antitumorT cell immunity".Aging 12.1 (2020):1-27.

25. HayflickLJECR. "The limited In vitro lifetime of human diploid cell strains". Experimental Cell Research37 (1965): 614-636.

26. Kuilman T andMichaloglou C. "The essence of senescence". Genes and Development 24 (2010): 2463-2479.

27. SlutskyR and Romero R. "Exhausted and Senescent T Cells at the Maternal-Fetal Interface in Preterm and Term Labor". Journal of Immunology Research (2019): 3128010.
28. Hernandez-Segura A and Nehme J. "Hallmarks of Cellular Senescence”. Trends in Cell Biology 28 (2018): 436-453.

29. Seluanov A andGorbunova V. "Change of the death pathway in senescent human fibroblasts in response to DNA damage is caused by an inability to stabilize p53". Molecular and Cellular Biology 21 (2001): 1552-1564.

30. Zhang J and Patel JM. "Enhanced apoptosis in prolonged cultures of senescent porcine pulmonary arteryendothelial cells". Mechanisms of Ageing and Development 123 (2002): 613-625.

31. WatanabeS and Kawamoto S. "Impact of senescence-associated secretory phenotype and its potential as a therapeutic target for senescence-associated diseases". Cancer Science 108 (2017): 563-569.

32. Roche A and Ross E. "Representative literature on the phytonutrients category: Phenolic acids". Critical Reviews in Food Science and Nutrition 57 (2017): 1089-1096.

33. JiS and Zheng Z. "Resveratrol promotes oxidative stress to drive DLC1 mediated cellular senescence in cancer cells". EXperimental Cell Research 370 (2018): 292-302.

34. TeponnoRB andKusariS. "Recent advances in research on lignans and neolignans". Natural Product Reports 33 (2016): 1044-1092.

35. MalekiSJ and Crespo JF. "Anti-inflammatory effects of flavonoids". Food Chemistry 299 (2019): 125124.

36. Zamin LL and Filippi-ChielaEC. "Resveratrol and quercetin cooperate to induce senescence-like growth arrest in C6 rat glioma cells". Cancer Science 100 (2009): 1655-1662.

37. Filippi-ChielaEC andThome MP. "Resveratrol abrogates the temozolomide-induced G2 arrest leading to mitotic catastrophe and reinforces the temozolomide-induced senescence in glioma cells". BMC Cancer 13 (2013): 147.

38. Sprouse AA and Herbert BS. "Resveratrol augments paclitaxel treatment in MDA-MB-231 and paclitaxel-resistant MDAMB-231 breast cancer cells". Anticancer Research 34 (2014): 5363-5374.

39. Zhu Y and HeW. "Resveratrol overcomes gefitinib resistance by increasing the intracellular gefitinib concentration and triggering apoptosis, autophagy and senescence in PC9/G NSCLC cells". Scientific Reports 5 (2015): 17730.

40. Luo $\mathrm{H}$ and WangL. "Resveratrol enhances ionizing radiationinduced premature senescence in lung cancer cells". International Journal of Oncology 43 (2013): 1999-2006. 
41. BhardwajA and Sethi G. "Resveratrol inhibits proliferation, induces apoptosis, and overcomes chemoresistancethrough down-regulation of STAT3 and nuclear factor-kappaB-regulated antiapoptotic and cell survival gene products in human multiple myeloma cells". Blood 109 (2007): 2293-2302.

42. SongNR and Chung MY. "Quercetin suppresses invasion and migration of H-Ras-transformed MCF10Ahuman epithelial cells by inhibiting phosphatidylinositol 3-kinase". Food Chemistry142 (2014): 66-71.

43. GuoY and Ayers JL. "Senescence-associated tissue microenvironment promotes colon cancer formation throughthe secretory factor GDF15". Aging Cell 18 (2019): e13013.

44. Menicacci B andMargheriF. "Chronic Resveratrol Treatment Reduces the Pro-angiogenic E_ect of HumanFibroblast "Senescent-Associated Secretory Phenotype" on Endothelial Colony-Forming Cells: The Role ofIL8". Journals of Gerontology Series A: Biological Sciences and Medical Sciences 74 (2019): 625-633.

45. PerrottKM and WileyCD. "Apigenin suppresses the senescence-associated secretory phenotype and paracrine effects on breast cancer cells". Geroscience39 (2017): 161-173.

46. LiuS and Zheng Z. "Resveratrol reduces senescence-associated secretory phenotype by SIRT1/NF-kappaBpathway in gut of the annual fish Nothobranchiusguentheri". Fish and Shellfish Immunology80 (2018): 473-479.

47. Serrano M and Lin AW. "Oncogenic ras provokes premature cell senescence associated with accumulation ofp53 and p16INK4a". Cell 88 (1997): 593-602.

48. PanHC and Jiang Q. "Quercetin promotes cell apoptosis and inhibits the expression of MMP-9 and fibronectin via the AKT and ERK signalling pathways in human glioma cells". Neurochemistry International 80 (2015): 60-71.

49. RusinM andZajkowicz, A. "Resveratrol induces senescencelike growth inhibition of U-2 OS cells associatedwith the instability of telomeric DNA and upregulation of BRCA1". Mechanisms of Ageing and Development 130 (2009): 528-537.

50. Bian Y.,etal. "Natural phenolstargeting senescence:a novel prevention and therapy of cancer".International Journal of Molecular Sciences21 (2020):684.

\section{Assets from publication with us}

- Prompt Acknowledgement after receiving the article

- Thorough Double blinded peer review

- Rapid Publication

- Issue of Publication Certificate

- High visibility of your Published work

Website: https://www.actascientific.com/

Submit Article: https://www.actascientific.com/submission.php Email us: editor@actascientific.com

Contact us: +919182824667 